Pacific Journal of Mathematics

APPROXIMATION OF IMPROPER INTEGRALS BY SUM

R. SHe nt 


\title{
APPROXIMATION OF IMPROPER INTEGRALS BY SUMS OVER MULTIPLES OF IRRATIONAL NUMBERS
}

\author{
R. SHERMAN LEHMAN
}

1. Introduction and notation. Let $\alpha$ be a positive irrational number. The multiples of $\alpha$, the numbers $\alpha, 2 \alpha, 3 \alpha, \ldots$, are equidistributed mod 1. Suppose $f(x)$ is a bounded function, Riemann integrable on the interval $(0,1)$, and periodic with period 1. It follows from Weyl's theory of equidistribution [2] that

$$
\lim _{N \rightarrow \infty} \frac{1}{N} \sum_{n=1}^{N} f(n \alpha)=\int_{0}^{1} f(x) d x .
$$

The purpose of this paper is to determine what modifications of this result are required when $f(x)$ is improperly Riemann integrable.

Every positive irrational number $\alpha$ has an infinite continued fraction expansion,

$$
\alpha=b_{0}+\frac{1}{b_{1}+\frac{1}{b_{2}+}+\frac{1}{b_{3}+\cdots,},}
$$

where the $b_{i}$ are integers such that $b_{0} \geq 0$, and $b_{i}>0$ for $i=1,2,3, \ldots$. Let $p_{i} / q_{i}(i=0,1,2, \ldots)$ be the convergents to $\alpha$. The integers $p_{i}$ and $q_{i}$ are relatively prime, and

$$
\begin{aligned}
& p_{0}=b_{0}, \quad q_{0}=1, \\
& p_{1}=b_{1} b_{0}+1, \quad q_{1}=b_{1}, \\
& p_{i+1}=b_{i+1} p_{i}+p_{i-1}, \\
& q_{i+1}=b_{i+1} q_{i}+q_{i-1}, \\
& p_{i} q_{i-1}-q_{i} p_{i-1}=(-1)^{i-1}
\end{aligned}
$$$$
(i=1,2,3, \ldots) \text {. }
$$

Received June 5, 1953. Presented to the Society, May 2, 1953. This work was performed under a contract with the Office of Naval Research.

Pacific J. Math. 5 (1955), 93- 102 
Let $\Delta_{i}=\alpha-p_{i} / q_{i}$. We shall make use of the inequalities

$$
(-1)^{i} \Delta_{i}>0 \text { and }\left|\Delta_{i}\right|<\frac{1}{q_{i} q_{i+1}}<\frac{1}{b_{i+1} q_{i}^{2}} .
$$

In our discussion we shall often omit the subscript $i$; thus $q=q_{i}, \Delta=\Delta_{i}$.

Denote by $R(y)$ the fractional part of $y$, often expressed by the notation $y-[y]$.

2. Theorem. We shall establish the following result.

THEOREM 1. Suppose $f(x)$ is a periodic function with period 1 , improperly Riemann integrable on the interval $(0,1)$, and bounded in every closed subinterval which excludes the points 0 and 1 . Suppose further that $f(x)$ is bounded or monotone near 0 and bounded or monotone near 1 in $(0,1)$. Then, for $N \rightarrow \infty$,

$$
\frac{1}{N} \sum_{n=1}^{N} f(n \alpha)=\int_{0}^{1} f(x) d x+\frac{1}{N} \sum_{k=1}^{b} f\left(k q_{i} \alpha\right)+o(1),
$$

where $N=b q_{i}+r, q_{i} \leq N<q_{i+1}$, and $0 \leq r<q_{i}$.

REMARKS. 1. We note that the theorem includes the case in which

$$
\lim _{x \rightarrow 0^{+}} f(x)=+\infty \text { and } \lim _{x \rightarrow 1_{-}^{-}} f(x)=-\infty
$$

The conclusion of the theorem can also be stated in the form

$$
\lim _{N \rightarrow \infty} \frac{1}{N} \sum_{\substack{n \leq N \\ q_{i} \backslash \bar{n}}} f(n \alpha)=\int_{0}^{1} f(x) d x,
$$

where the sum is extended over the positive integers $n \leq N$ which are not multiples of $q_{i}$, where $q_{i}$ is the largest denominator of a convergent to $\alpha$ that is less than or equal to $N$. Observe that the values of $n$ which are not summed over are independent of the particular function $f(x)$.

2. This theorem contains a result proved by MacMillan [1], who showed that if $D(y)$ is the absolute value of the difference between $y$ and the nearest integer, so that $0 \leq D(y) \leq 1 / 2$, and $\alpha$ is an irrational number for which 


$$
\lim _{i \rightarrow \infty} b_{i+1}^{-1 / q_{i}}=1
$$

then

$$
\lim _{N \rightarrow \infty} \frac{1}{N} \sum_{n=1}^{N} \log D(n \alpha)=-\log 2 e=\int_{-1 / 2}^{1 / 2} \log |x| d x .
$$

Proof. First, consider the case that $|f(x)|$ is bounded. If $|f(x)| \leq M$, we have

$$
\left|\frac{1}{N} \sum_{k=1}^{b} f(k q \alpha)\right| \leq b \frac{M}{N} \leq \frac{M}{q}
$$

which tends to zero as $N \longrightarrow \infty$. It follows from this and Weyl's result that the conclusion (1) is valid in the special case that $|f(x)|$ is bounded.

It will be sufficient to prove the theorem in the case that for $x$ near $0,|f(x)|$ is monotonely decreasing as $x$ increases; and for $x$ near 1 is monotonely increasing in $(0,1)$. Certainly $f(x)$ can be written as the sum of a function with these additional properties and a bounded integrable function. Now, (1) is valid for a bounded integrable function; and further, if the equation (1) holds for two functions, it must hold for their sum. Therefore, we may limit our considerations as indicated.

Decompose $f(x)$ into three periodic functions $f_{\epsilon}(x), g_{\epsilon}(x)$, and $h_{\epsilon}(x)$ of period 1 , where $\epsilon$ is a small positive parameter to be chosen later, with

$$
\begin{array}{ll}
f_{\epsilon}(x)=f(x) & \text { for } \epsilon<x<1-\epsilon, \\
f_{\epsilon}(x)=0 & \text { for } x \leq \epsilon \text { or } x \geq 1-\epsilon, \\
g_{\epsilon}(x)=f(x) \text { for } x \leq \epsilon, \\
g_{\epsilon}(x)=0 \quad \text { for } x>\epsilon, \\
h_{\epsilon}(x)=f(x) \text { for } x \geq 1-\epsilon, \\
h_{\epsilon}(x)=0 \quad \text { for } x<1-\epsilon .
\end{array}
$$

We define $A$ as the set composed of the positive integers $n \leq N$ for which

$$
\frac{1}{q}<R(n \alpha)<1-\frac{1}{q}
$$


Then, for $N$ sufficiently large, we have

$$
\frac{1}{N} \sum_{n \in A} f(n \alpha)=\frac{1}{N}\left\{\sum_{n=1}^{N} f_{\epsilon}(n \alpha)+\sum_{n \in A} g_{\epsilon}(n \alpha)+\sum_{n \in A} h_{\epsilon}(n \alpha)\right\}
$$

By Weyl's result,

$$
\lim _{N \rightarrow \infty} \frac{1}{N} \sum_{n=1}^{N} f_{\epsilon}(n \alpha)=\int_{0}^{1} f_{\epsilon}(x) d x=\int_{\epsilon}^{1-\epsilon} f(x) d x
$$

Since $p$ and $q$ are relatively prime, when $n$ runs through the integers 1,2 , $3, \cdots, q, R(n p / q)$ runs through the numbers $m / q(m=0,1,2, \cdots, q-1)$, taking on each value exactly once. Therefore, when $n$ runs through the integers 1,2 , $3, \cdots, N=b q+r$, each of the values $m / q$ is assumed by $R(n p / q)$ exactly $b$ times if $r=0$; and if $r \neq 0$ each value $m / q$ is assumed either $b$ or $(b+1)$ times. But since

$$
R(n \alpha)=R\left(n \frac{p}{q}+n \Delta\right)
$$

where

$$
0<(-1)^{i}{ }_{n} \Delta<\frac{n}{q_{i} q_{i+1}} \leq \frac{N}{q_{i} q_{i+1}}<\frac{1}{q}
$$

it also is true that the intervals $(m / q,(m+1) / q)$ each contain $b$ values of $R(n \alpha)$ if $r=0$, and either $b$ or $(b+1)$ values if $r \neq 0$.

We choose $\epsilon$ sufficiently small so that $\left|g_{\epsilon}(x)\right|$ and $\left|h_{\epsilon}(x)\right|$ are monotone functions in $(0,1)$. Then if we compare $g_{\epsilon}(n \alpha)$ with $g_{\epsilon}(m / q)$ when $R(n \alpha)$ lies in the interval $(m / q,(m+1) / q)$ we have $\left|g_{\epsilon}(n \alpha)\right| \leq\left|g_{\epsilon}(m / q)\right|$. Hence

$$
\begin{aligned}
\left|\frac{1}{N} \sum_{n \in A} g_{\epsilon}(n \alpha)\right| \leq \frac{1}{b q} \sum_{m=1}^{q-2}(b+1)\left|g_{\epsilon}\left(\frac{m}{q}\right)\right| & \leq 2 \int_{0}^{1-2 / q}\left|g_{\epsilon}(x)\right| d x \\
& =2 \int_{0}^{\epsilon}|f(x)| d x,
\end{aligned}
$$

since $q$ is large. Similarly, when $R(n \alpha)$ lies in $(m / q,(m+1) / q)$ we have

$$
\left|h_{\epsilon}(n \alpha)\right| \leq\left|h_{\epsilon}\left(\frac{m+1}{q}\right)\right| \text {, }
$$


and hence

$$
\begin{aligned}
\left|\frac{1}{N} \sum_{n \in A} h_{\epsilon}(n \alpha)\right|<\frac{1}{b q} \sum_{m=1}^{q-2}(b+1)\left|h_{\epsilon}\left(\frac{m+1}{q}\right)\right| & \leq 2 \int_{2 / q}^{1}\left|h_{\epsilon}(x)\right| d x \\
& =2 \int_{1-\epsilon}^{1}|f(x)| d x .
\end{aligned}
$$

It now follows from (2) and (3) that

$$
\left|\frac{1}{N} \sum_{n \in A} f(n \alpha)-\int_{0}^{1} f(x) d x\right| \leq 3 \int_{0}^{\epsilon}|f(x)| d x+3 \int_{1-\epsilon}^{\epsilon}|f(x)| d x+o(1)
$$

as $N \rightarrow \infty$. Letting $\epsilon$ tend to 0 , we find

$$
\frac{1}{N} \sum_{n \in A} f(n \alpha)=\int_{0}^{1} f(x) d x+o(1)
$$

as $N \rightarrow \infty$.

Now, we must consider the terms of (1) for which the $R(n \alpha)$ are in (1$1 / q, 1)$ or $(0,1 / q)$. There are different cases depending on whether $i$ is even or odd. We suppose first that $i$ is even. In this case we shall find that the sum of the terms for which $R(n \alpha)$ is in $(1-1 / q, 1)$ is $o(1)$. The sum of terms for which $R(n \alpha)$ is in $(0,1 / q)$, on the other hand, is not necessarily $o(1)$. This accounts for the additional terms beside the integral in the right side of (1).

We first want to find all $n \leq N$ for which the $R(n \alpha)$ are in $(1-1 / q), 1)$. Since $i$ is even, we have

$$
\begin{gathered}
p_{i} q_{i-1}-q_{i} p_{i-1}=-1, \\
0<n \Delta_{i}<\frac{1}{q_{i}},
\end{gathered}
$$

and therefore $R\left(q_{i-1} p / q\right)=1-1 / q$. Then, since $n \alpha=n p / q+n \Delta, R(n \alpha)$ will be in $(1-1 / q, 1)$ if and only if $R(n p / q)=1-1 / q$. Repeating the reasoning preceding formula $(4)$, we observe that necessarily $n \equiv q_{i-1}(\bmod q)$.

We define $b^{\prime}=b-1$ if $r<q_{i-1}$ and $b^{\prime}=b$ if $r \geq q_{i-1}$. Further, $r$ can be $\geq q_{i-1}$ only in case $b<b_{i+1}$, for 


$$
N=b q_{i}+r<q_{i+1}=b_{i+1} q_{i}+q_{i-1}
$$

by assumption. Hence in any case we have $b^{\mathcal{D}} \leq b_{i+1}-1$.

Thus $R(n \alpha)$ is in $(1-1 / q, 1)$ if and only if

$$
n=k q+q_{i-1} \quad\left(k=0,1,2, \cdots, b^{\prime}\right)
$$

and

$$
R\left(\left(k q+q_{i-1}\right) \alpha\right)=1-\frac{1}{q}+k q \Delta+q_{i-1} \Delta
$$

Now let

$$
S=\frac{1}{N} \sum_{R(n \alpha)>1-1 / q} f(n \alpha)
$$

Because of the monotonicity of $|f(x)|$ near 1 , we have

$$
|q \Delta \cdot S|=\frac{1}{N} \sum_{k=0}^{b^{\prime}}\left|f\left(1-\frac{1}{q}+k q \Delta+q_{i-1} \Delta\right)\right| \cdot q \Delta \leq \frac{1}{N} \int_{\lambda}^{\mu}|f(x)| d x,
$$

where

$$
\lambda=1-\frac{1}{q}+q_{i-1} \Delta, \mu=1-\frac{1}{q}+\left(b^{\circ}+1\right) q \Delta+q_{i-1} \Delta .
$$

Also $\mu<1$ because $b^{\prime} \leq b_{i+1}-1$, and hence

$$
\left(b^{\prime}+1\right) q \Delta+q_{i-1} \Delta \leq\left(b_{i+1} q+q_{i-1}\right) \Delta=q_{i+1} \Delta<\frac{q_{i+1}}{q q_{i+1}}=\frac{1}{q} .
$$

By assumption the function $|f(x)|$ is monotonely increasing in the interval $(1-1 / q, 1)$ for $q$ sufficiently large. Its average over the interval $(\lambda, \mu)$ must be less than its average over an interval closer to 1 . From this it follows that

$$
\int_{\lambda}^{\mu}|f(x)| d x \leq \frac{\mu-\lambda}{1-\lambda} \int_{\lambda}^{1}|f(x)| d x=\frac{\left(b^{\prime}+1\right) q \Delta}{(1 / q)-q_{i-1}} \int_{\lambda}^{1}|f(x)| d x .
$$

But 


$$
\frac{1}{q}-q_{i-1} \Delta>\frac{1}{q}-\frac{q_{i-1}}{q q_{i+1}}=\frac{q_{i+1}-q_{i-1}}{q q_{i+1}}>\frac{b_{i+1} q}{q\left(b_{i+1}+1\right) q} \geq \frac{1}{2 q}
$$

since $b_{i+1} \geq 1$. Hence

$$
\begin{aligned}
& |S| \leq \frac{2 q\left(b^{\prime}+1\right) q \Delta}{(b q+r) q \Delta} \int_{\lambda}^{1}|f(x)| d x \leq \frac{2 q(b+1)}{b q} \int_{\lambda}^{1}|f(x)| d x, \\
& |S| \leq 4 \int_{\lambda}^{1}|f(x)| d x .
\end{aligned}
$$

Since $\lambda \geq 1-1 / q$, which tends to 1 as $N \rightarrow \infty, S \rightarrow 0$ as $N \rightarrow \infty$.

The only terms of (1) which we have not considered yet are those for which the $R(n \alpha)$ lie in $(0,1 / q)$. But $R(n \alpha)$ will be in $(0,1 / q)$ if and only if $R(n p / q)=0$ which occurs only when $n=k q(k=1,2,3, \ldots, b)$. Thus we find

$$
\frac{1}{N} \sum_{R(n \alpha)<1 / q} f(n \alpha)=\frac{1}{N} \sum_{k=1}^{b} f(k q \alpha) \text {. }
$$

Using (5) we have finally

$$
\frac{1}{N} \sum_{n=1}^{N} f(n \alpha)=\int_{0}^{1} f(x) d x+\frac{1}{N} \sum_{k=1}^{b} f(k q \alpha)+o(1)
$$

for $N \rightarrow \infty$.

We have assumed that $i$ is even, but the case in which $i$ is odd is similar and can be treated in a corresponding manner. In this case, however, it is the sum of the terms for which $R(n \alpha)$ is in $(0,1 / q)$ which is $o(1)$, and the terms for which $R(n \alpha)$ is in $(1-1 / q, 1)$ which account for the additional terms beside the integral in the right side of (1). The result obtained is the same as (6). The proof of Theorem 1 is then complete.

3. Further results. We start with two remarks.

1. It is not difficult to determine for what choice of $N$ for $q_{i} \leq N<q_{i+1}$ the sum term in the right side of formula ( 1 ) is largest. We have

$$
\left|\frac{1}{N} \sum_{k=1}^{b} f\left(k q_{i} \alpha\right)\right|=\left|\frac{1}{b q_{i}+r} \sum_{k=1}^{b} f\left(k q_{i} \Delta\right)\right| \leq\left|\frac{1}{q_{i}} f\left(q_{i} \Delta_{i}\right)\right| .
$$


We see that the sum term is largest in absolute value when $N=q_{i}$, the denominator of a continued fraction convergent, for then

$$
\frac{1}{q_{i}} \sum_{n=1}^{q_{i}} f(n \alpha)=\int_{0}^{1} f(x) d x+\frac{1}{q_{i}} f\left(q_{i} \Delta_{i}\right)+o(1) .
$$

On the other hand, it follows from this that if $N=q_{i}-1$,

$$
\frac{1}{q_{i}-1} \sum_{n=1}^{q_{i}-1} f(n \alpha)=\frac{q_{i}}{q_{i}-1} \int_{0}^{1} f(x) d x+o(1)=\int_{0}^{1} f(x) d x+o(1) .
$$

Thus, we see Weyl's result holds without omitting terms from the sum if $N \rightarrow \infty$ over the sequence of numbers $q_{1}-1, q_{2}-1, q_{3}-1, \ldots$ MacMillan [1] proved this result in the case

$$
f(x)=\log |x| \text { for }-\frac{1}{2} \leq x \leq \frac{1}{2} .
$$

2. If there exists a constant $c>0$ such that

$$
\left|\Delta_{i}\right|>\frac{1}{c q_{i}^{2}}
$$

for all $q_{i}$, then

$$
\left|\frac{1}{q} f(q \Delta)\right| \leq\left|\frac{1}{q} f\left(\frac{1}{c q}\right)\right| \leq c \int_{0}^{1 / c q}|f(x)| d x
$$

which tends to 0 as $q \longrightarrow \infty$. Such a constant $c$ exists if the quotients $b_{k}$ of the continued fraction for $\alpha$ are bounded.

THEOREM 2. For all irrational $\alpha>0$ with the property that the quotients of the continued fraction for $\alpha$ are bounded,

$$
\lim _{N \rightarrow \infty} \frac{1}{N} \sum_{n=1}^{N} f(n \alpha)=\int_{0}^{1} f(x) d x
$$

In particular this formula holds for all $\alpha$ which are quadratic irrationals.

For almost all $\alpha$ Weyl's result holds without omitting terms from the sum. 
THEOREM 3. Suppose $f(x)$ satisfies the hypothesis of Theorem 1. Then, for almost all $\alpha$,

$$
\lim _{N \rightarrow \infty} \frac{1}{N} \sum_{n=1}^{N} f(n \alpha)=\int_{0}^{1} f(x) d x .
$$

Proof. From Theorem 1 and (7) one can see that it will be sufficient to prove that

$$
\frac{1}{n} f(n \alpha) \rightarrow 0
$$

as $n \rightarrow \infty$, for almost all $\alpha$.

Let $E_{k}$ be the set of $\alpha$ for which $0<\alpha \leq 1$ and $|f(\alpha)|>k / \nu$, where $\nu$ is a positive integer. Interpreting $\int_{0}^{1}|f(x)| d x$ as a Lebesgue integral we have

$$
\frac{1}{\nu} \sum_{k=1}^{\infty} m\left(E_{k}\right) \leq \int_{0}^{1}|f(x)| d x,
$$

where $m\left(E_{k}\right)$ is the measure of $E_{k}$. The measure of $E_{k}$ is equal to $1 / k$ times the measure of the set of $\alpha$ for which

$$
0<\alpha \leq k \text { and } \frac{1}{k}|f(\alpha)|>\frac{1}{v}
$$

because of the periodicity of $f(x)$. It follows that $m\left(E_{k}\right)=m\left(F_{k}\right)$, where $F_{k}$ is the set of $\alpha$ for which

$$
0<\alpha \leq 1 \text { and } \frac{1}{k}|f(k \alpha)|>\frac{1}{\nu}
$$

Hence $\sum_{k=1}^{\infty} m\left(F_{k}\right)$ converges.

Let $G_{\nu}$ be the set of all $\alpha$ for which $0<\alpha \leq 1$ and for which there are infinitely many $n$ such that

$$
\frac{1}{n}|f(n \alpha)|>\frac{1}{\nu}
$$

Thus $G_{\nu}$ is the set consisting of all $\alpha$ which belong to an infinite number of the sets $F_{1}, F_{2}, F_{3}, \ldots$. Any open set which covers the union of the sets 
$F_{k}, F_{k+1}, F_{k+2}, \cdots$ will also cover $G_{\nu}$ no matter how large $k$ is. But this union will have arbitrarily small measure if $k$ is chosen sufficiently large, because $\sum_{i=1}^{\infty} m\left(F_{i}\right)$ converges. It follows that $m\left(G_{\nu}\right)=0$.

Now let $G$ be the set of all $\alpha$ for which $0<\alpha \leq 1$ and $f(n \alpha) / n$ does not tend to 0 as $n \rightarrow \infty$. The set $G$ is the union of the sets $G_{1}, G_{2}, G_{3}, \ldots$. Since each of the sets $G_{\nu}$ has measure zero, $G$ has measure 0 , which was to be proved.

If $f(x)$ becomes infinite at points other than the end points, a result which corresponds to $(5)$ can be proved. Again the sum tends to the integral provided certain terms are omitted. The terms omitted depend on the positions of the singularities of $f(x)$ but otherwise do not depend on the particular function $f(x)$. We omit the proof of the following theorem because it is similar to the proof of (5).

THE OREм 4. Suppose $f(x)$ is a periodic function with period 1, improperly Riemann integrable on the interval $(0,1)$, and bounded except in the neighborhood of a finite number of exceptional points in the interior of $(0,1)$. Suppose, furthermore, that in the half-neighborhood to the right of an exceptional point $X, f(x)$ is bounded or monotone; and also in the half-neighborhood to the left of $X, f(x)$ is bounded or monotone. Let $N=b q_{i}+r, q_{i} \leq N<q_{i+1}$, and define the set $A$ composed of positive integers $\leq N$ in the following way. If $R(n \alpha)$ is in the interval $(m / q,(m+1) / q)$ put $n$ in $A$ if and only if there is no exceptional point $X$ such that $(m-1) / q<X<(m+2) / q$. Then

$$
\lim _{N \rightarrow \infty} \frac{1}{N} \sum_{n \in A} f(n \alpha)=\int_{0}^{1} f(x) d x .
$$

\section{REFERENCES}

1. W. D. MacMillan, A theorem connected with irrational numbers, Amer. J. Math. 38 (1916), 387 - 396.

2. H. Weyl, Über die Gleichverteilung von Zahlen mod. Eins, Math. Ann. 77 (1916), 313-352.

STANFORD UNIVERSITY 



\section{Pacific Journal of Mathematics}

\section{Vol. 5, No. $1 \quad$ September, 1955}

Frank Herbert Brownell, III, Flows and noncommuting projections on

Hilbert space ................................... 1

H. E. Chrestenson, A class of generalized Walsh functions ............. 17

Jean Bronfenbrenner Crockett and Herman Chernoff, Gradient methods of maximization ................................... 33

Nathan Jacob Fine, On groups of orthonormal functions. I .......... 51

Nathan Jacob Fine, On groups of orthonormal functions. II ............ 61

Frederick William Gehring, A note on a paper by L. C. Young .......... 67

Joachim Lambek and Leo Moser, On the distribution of Pythagorean

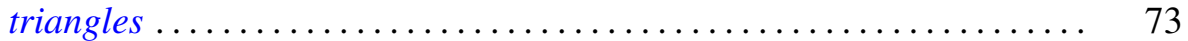

Roy Edwin Wild, On the number of primitive Pythagorean triangles with

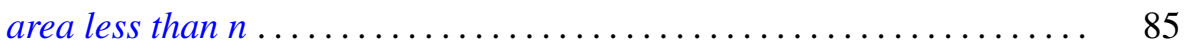

R. Sherman Lehman, Approximation of improper integrals by sums over multiples of irrational numbers ........................ 93

Emma Lehmer, On the number of solutions of $u^{k}+D \equiv w^{2}(\bmod p) \ldots 103$

Robert Delmer Stalley, A modified Schnirelmann density............... 119

Richard Allan Moore, The behavior of solutions of a linear differential equation of second order............................. 125

William M. Whyburn, A nonlinear boundary value problem for second order differential systems. 